\section{Le paradoxe cardiovasculaire de la grossesse}

Jean St-Louis, Michèle Brochu
> Malgré l'accès plus généralisé aux soins prénataux dans les pays développés, on connaît très peu les mécanismes qui régissent l'adaptation maternelle à l'état physiologique qu'est la grossesse. Deux études récentes nous rappellent que certains phénomènes se manifestent très tôt lors de la grossesse et que le succès de leur mise en place assurerait la poursuite d'une grossesse en santé. Ces études concernent respectivement la fonction du corps jaune de gestation et le réajustement du système rénine - angiotensine - aldostérone (SRAA) favorisant les actions minéralocorticoïdes de l'angiotensine II aux dépens de ses effets vasculaires. Ainsi, malgré l'activation du SRAA, on observerait une vasodilatation périphérique importante et une augmentation compensatoire du volume sanguin. On présume que les stéroïdes sexuels joueraient un rôle dans ces adaptations hémodynamiques propres à la grossesse. <

\section{too ans \\ a taire grandir la vie.}

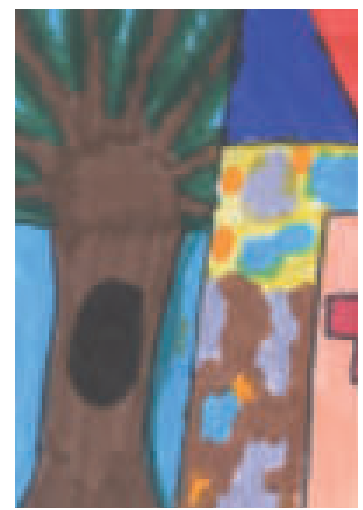

Centre de recherche, CHU Sainte-Justine,

Département d'obstétrique et gynécologie, Faculté de médecine, Université de Montréal, 3175, chemin de la Côte SainteCatherine, Montréal (Québec), H3T 1C5 Canada. jean.st-louis@umontreal.ca michele.brochu@umontreal.ca

qui a été, au cours des années, l'objet d'hypothèses relevant des courants qui ont traversé la science biomédicale. Ainsi, on l'aurait attribuée à une attaque oxydante, un processus inflammatoire, une mésadaptation hémodynamique ou un déséquilibre endocrinien ou métabolique, etc. [2]. Depuis une quinzaine d'années, un certain nombre de chercheurs estiment qu'une dysfonction endothéliale, résultant de la faillite de l'invasion trophoblastique des artères spiralées utérines, accompagnée d'une attaque oxydante, entraveraient le processus physiologique d'adaptation à la grossesse et mèneraient à la prééclampsie [2]. Ainsi, on a entrepris des essais cliniques destinés à prévenir la prééclampsie fondés sur l'ingestion de suppléments de vitamines C et $\varepsilon$ (anti-oxydants) [3]. II a fallu arrêter ces études, parce que les suppléments vitaminés provoquaient des effets indésirables sur le fœtus. C'est pourquoi nous devons reconsidérer les mécanismes de ce syndrome. On a avancé, par exemple, l'hypothèse selon laquelle des anticorps agonistes des récepteurs ATl de l'angiotensine, de concert avec le FLTl soluble (fms-like tyrosine kinase 1), joueraient un rôle dans la genèse de cette affection [4]. On a aussi attribué à une augmentation du volume sanguin, associée à un trouble de la gestion du sodium par le rein, la mobilisation d'inhibiteurs de la $\mathrm{Na} / \mathrm{K}$-ATPase (bufodiènolides) qui nuirait à l'invasion trophoblastique des artères spiralées [5]. Salas et al. [6] ont suggéré que chez la femme l'expansion retardée ou lente du volume sanguin et l'élévation rapide de la production de progestérone (antagoniste de l'aldostérone?) installeraient, avant le $2^{\mathrm{e}}$ trimestre, les conditions propres à provoquer au $3^{e}$ trimestre l'apparition 
des manifestations cliniques de la maladie. Ces perspectives nous ramènent sans contradiction au problème que pose la compréhension des mécanismes d'adaptations cardiovasculaires et hormonales précoces particuliers à la grossesse [7]. C'est peut-être la physiologie de la grossesse qui est «singulière », et non pas la prééclampsie!

\section{La grossesse normale}

En effet, la grossesse est un état physiologique singulier, qui a notamment la propriété d'instaurer un nouvel équilibre physiologique au sein de plusieurs systèmes, en particulier les systèmes cardiovasculaire, endocrinien et rénal. Dans d'autres circonstances, ces changements seraient pathologiques et constitueraient une menace pour la santé, comme, par exemple, les concentrations plasmatiques très élevées d'œstrogènes et de progestérone qui s'installent rapidement au cours de la grossesse ou encore la hausse impressionnante du volume sanguin. Celle-ci apparaît très tôt au cours de la grossesse [7] ; de plus, elle s'accompagne d'une augmentation du débit cardiaque et, paradoxalement, d'une baisse de la pression artérielle. Ce nouvel équilibre cardiovasculaire résulte d'une importante réduction de la résistance vasculaire périphérique, ce phénomène est d'ailleurs abondamment documenté. Les substances et les mécanismes qui conduisent à cette vasodilatation restent encore à identifier; néanmoins les composés les plus fréquemment considérés sont le monoxyde d'azote (NO) et son second messager, le guanosine 3',5' monophosphate cyclique (cGMP). Toutefois, les données disponibles, chez la femme enceinte comme chez les animaux de laboratoire durant la gestation, demeurent contradictoires [8], même si la vasodilatation dépendante de l'endothélium est la base du concept de «dysfonction endothéliale », comme étape pathogénique essentielle à la prééclampsie.

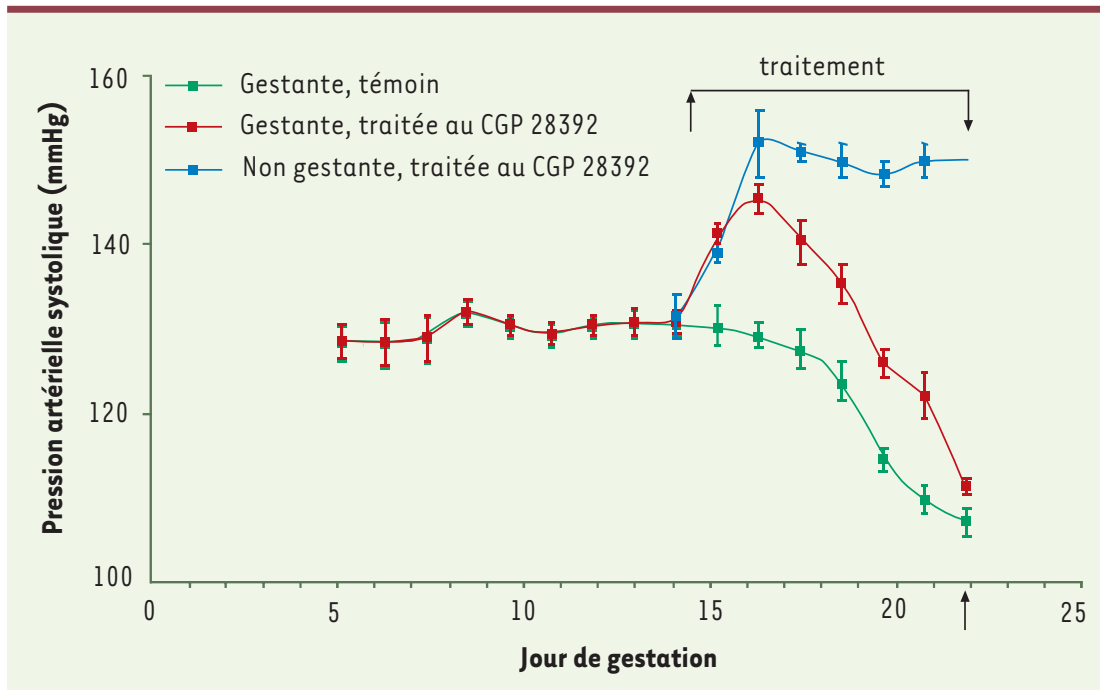

Figure 1. Profil de la pression artérielle systolique chez des rates gestantes traitées $(n=15)$ et non-traitées $(n=13)$ et non-gestantes traitées $(n=6)$ avec un activateur de canaux calciques de type L (CGP 28392). Le trait (en haut à droite) représente la période de traitement au CGP 38392. L'ordonnée décrit la pression artérielle systolique (en $\mathrm{mm} \mathrm{Hg}$ ) mesurée par pléthysmographie caudale (Tail Cuff) chez la rate consciente. L'abscisse montre les jours de gestation (la flèche indique la parturition) (adaptée de [15]).
Il y a une vingtaine d'années, nous avions avancé l'idée que l'état anti-hypertensif caractéristique de la grossesse tenait à une diminution de la résistance vasculaire périphérique liée à la résistance de l'appareil vasculaire maternel aux stimulus vasoconstricteurs [9]. Une étude longitudinale hémodynamique et hormonale menée auprès de 13 femmes du milieu de la phase folliculaire du cycle menstruel et jusqu'à la mi-grossesse appuie le concept selon lequel la vasodilatation survient très tôt au cours de la grossesse, avant même que ne s'achève le processus de placentation (<8 semaines) [7]. C'est aussi lors de cette période que le volume sanguin augmente rapidement et que le SRAA est activé. Certes, il nous faudrait mieux connaître la séquence de l'apparition de ces paramètres.

Durant la grossesse, une résistance à l'action vasoconstrictrice de l'angiotensine II se développe, encore qu'elle ne soit pas spécifique à ce peptide [10]. Cette résistance se manifeste aussi chez les animaux de laboratoire et on la retrouve même à l'échelle des vaisseaux sanguins isolés $[11,12]$. Il semble que la baisse de résistance vasculaire périphériques, la vasodilatation et la résistance artérielle aux stimulus vasoconstricteurs soient liées $[9,13]$. On a attribué ce phénomène au fait que le processus de couplage excitation-réponse était perturbé. Ainsi, dans les artères de rates gestantes, il se traduit par un ralentissement de la mobilisation du calcium extracellulaire via les canaux calciques dépendants du voltage, sans diminution de l'expression de la protéine du canal [1], 13]. Ce phénomène apparaît aussi dans la glomerulosa surrénalienne [14] et les lymphocytes (Simaan, St-Louis, Brochu, observation non publiée).

\section{Développement d'un modèle animal de prééclampsie}

Nous avons alors traité des rates gestantes avec un activateur des canaux calciques dépendants du voltage dans le but d'interférer avec la réduction de l'activité des canaux. Le traitement n'a pas empêché la chute de pression artérielle à la fin de la gestation chez la rate, quoique l'activateur (CGP 28392) ait provoqué une hausse soutenue de la pression artérielle chez la rate non gestante (Figure 1) [15]. Ces résultats indiquent que la réduction de l'activité des canaux calciques n'occupe guère que 
la fonction d'un médiateur distal d'altérations plus précoces du système de couplage excitation - réponse dans les cellules musculaires lisses. Les résultats de certaines études suggèrent qu'il y aurait une altération du potentiel membranaire dans ces cellules $[16,17]$ ce qui concorde avec l'observation [18] d'une hyperpolarisation (7 mV) des cellules musculaires lisses mésentériques de rates gestantes comparées à des non-gestantes.

Pour tenter d'empêcher la baisse de pression artérielle qui accompagne la gestation, nous avons modulé l'activation du SRAA en modifiant l'ingestion de sodium. Nous avons administré une diète réduite en sodium ( 0,03 plutôt que $0,23 \%, p / p$, eau déminéralisée comme breuvage) durant les 7 derniers jours de gestation (sur 22) de la rate. Nous avons observé des augmentations très importantes de la PRA (activité rénine plasmatique) et de l'aldostérone plasmatique, accompagnées d'une légère accentuation de la chute de la pression artérielle. De plus, une restriction de la croissance a été observée chez les fœtus de 22 jours [19]. Ces données contrastent avec celles montrant qu'un régime sans sodium, commencé 7 jours avant le croisement, entraîne une hausse du volume sanguin à 12 jours de gestation, comme chez les rates gestantes sous régime normal ; l'étude n'ayant pas été poursuivie au delà du $12^{\mathrm{e}}$ jour de gestation [20]. Ces données suggèrent que la perturbation de l'homéostasie du sodium a des conséquences hémodynamiques plus importantes si elle est appliquée durant la gestation plutôt qu'avant.

Lorsque nous avons administré un supplément sodique $(\mathrm{NaCl} 0,9 \%$ ou $1,8 \%, \mathrm{p} / \mathrm{v}$, comme breuvage) pour les 7 derniers jours de gestation, nous avons observé une réduction, proportionnelle à la surcharge sodique, de la PRA et de l'aldostérone plasmatique. Les rates soumises au supplément de $\mathrm{NaCl}$ 0,9\% n'ont pas présenté la baisse de la pression artérielle caractéristique de la gestation tandis que celles soumises à la plus forte supplémentation $(1,8 \%)$ ont accusé une hausse de pression artérielle (Figure 2A) [21]. La résistance, particulière à la gestation, aux effets vasculaires de la phényléphrine, de la vasopressine et du chloment de [21] et de [22]). rure de potassium est abolie par le traitement des rates gestantes avec le supplément sodique de 0,9\% (Figure 2B) [22]. Nous estimons avoir établi ainsi un modèle d'hypertension gravidique expérimentale.

lanosi-Irimie et al. [5] ont administré à des rates de la désoxycorticostérone (DOCA) avec du $\mathrm{NaCl} 0,9 \%$ comme breuvage, avant leur croisement. Ils ont observé une hausse graduelle de la pression artérielle au cours de la gestation en comparaison avec des rates non traitées qui présentent la chute de la pression de fin de gestation. Ils ont aussi noté une baisse du volume sanguin (hausse de l'hématocrite) et une réduction du nombre de fœtus et du poids de la portée en comparaison avec les rates témoins. II nous semble qu'un tel traitement compromet considérablement l'intégrité fœtale, ce modèle s'apparentant davantage à une hypertension chronique qu'à une hypertension gestationnelle [9]. Nous n'avons pas observé d'indice d'une expansion volumique au-delà de celle observée chez la rate gestante normale [21] et le nombre de fœtus était inchangé. L'ensemble de ces données nous amène à considérer que la gestation provoque un réajustement

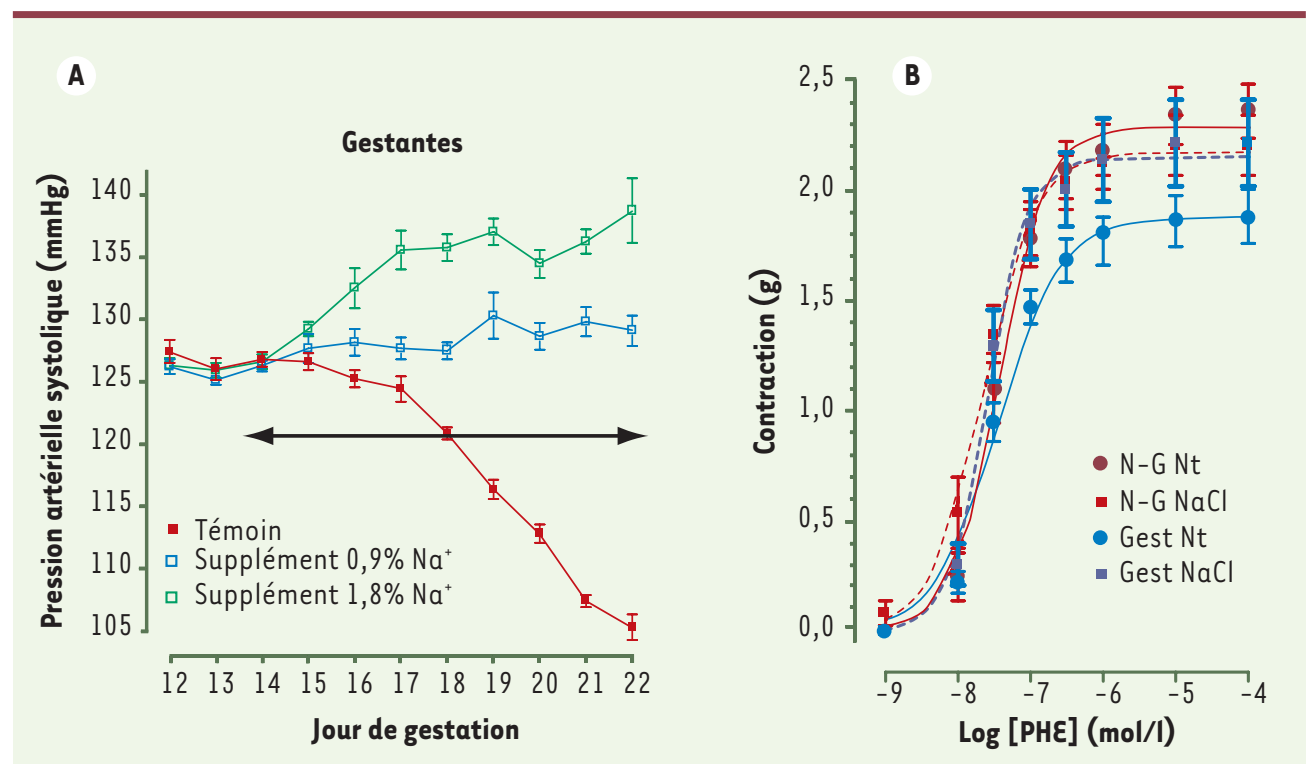

Figure 2. A. Effets de suppléments sodiques sur la pression systolique de rates en gestation au cours des jours 12 à 22 (terme $23^{e}$ jour). Les suppléments de 0,9 et $1,8 \% \mathrm{NaCl}(\mathrm{p} / \mathrm{v})$ ont été administrés comme breuvage à partir $\mathrm{du} 14^{\mathrm{e}}$ jour de gestation, comme l'indique la double flèche. $B$. Courbes concentration-réponse à la phényléphrine sur des anneaux d'aortes isolées de rates non-gestantes ( $N-G$, en rouge) et gestantes (Gest, en bleu). Les rates ont reçu ou non (respectivement, $\mathrm{NaCl}$ et $\mathrm{Nt}$ [non-traitées]) un supplément sodique de $0,9 \%$ (comme dans la Figure $2 A$ ) et ont été sacrifiées après 7 jours de traitement pour obtenir les aortes thoraciques. L'ordonnée décrit les réponses contractiles (en gramme-tension) obtenues après l'application, dans chaque bain contenant un anneau aortique, de concentrations croissantes de phényléphrine (PHE, abscisse). Chaque courbe représente la moyenne des réponses ( \pm e.s.) obtenues pour 9 anneaux aortiques de différents animaux (adaptées respective- 
des effets physiologiques normalement exercés par le SRAA. II est possible que l'action de l'angiotensine II sur la régulation hydro-sodée (via l'aldostérone) soit favorisée au détriment de ses effets vasculaires. Serait-il possible que l'absence de ce mécanisme soit une cause de la prééclampsie?

\section{Mécanismes d'adaptation proposés}

Selon les résultats obtenus chez la femme [7], il faut supposer que le premier stimulus d'adaptation à la grossesse soit hormonal et lié à l'activation du SRAA, par suite de la vasodilatation généralisée instaurée durant la phase lutéale de la grossesse, phase dépendante du corps jaune. Il est reconnu que les hormones de l'ovaire exercent des effets vasodilatateurs, ce phénomène est certes mieux documenté pour les œstrogènes que pour la progestérone (voir [23]). Ces facteurs provoquent une hausse graduelle du volume plasmatique; et le débit cardiaque (volume d'éjection systolique et fréquence) augmente alors graduellement sans doute en réponse à cette vasodilatation pour maintenir la pression artérielle. Vers la fin du premier trimestre, ces mécanismes compensatoires ne suffisent plus et la pression artérielle diminue alors que la dilatation périphérique continue à augmenter, comme l'illustre la Figure 3.

Durant la grossesse, l'organisme s'adapte constamment pour satisfaire les besoins croissants du fœtus. Certains de ces ajustements ont été clairement définis et décrits (reliés par un trait plein, Figure 4). Dans le système nerveux, un réajustement de la sécrétion neurohypophysaire d'hormone antidiurétique $(\mathrm{ADH})$ en fonction de l'osmolalité plasmatique [24], combiné à des augmentations de filtration glomérulaire et de rétention sodique, provoque une augmentation du volume sanguin. L'augmentation de ce dernier étant plus importante que la rétention sodée, l'activité du SRAA est accrue, entraînant une hausse de la sécrétion d'aldostérone, augmentant encore la rétention sodique. Ces changements devraient conduire à une hausse de la pression artérielle, mais ici, ils sont associés à une baisse de la pression, d'où le paradoxe de la grossesse (boîte bleue et lignes en tirets, Figure 4). D'autre part, la diminution de la sensibilité aux vasoconstricteurs et la

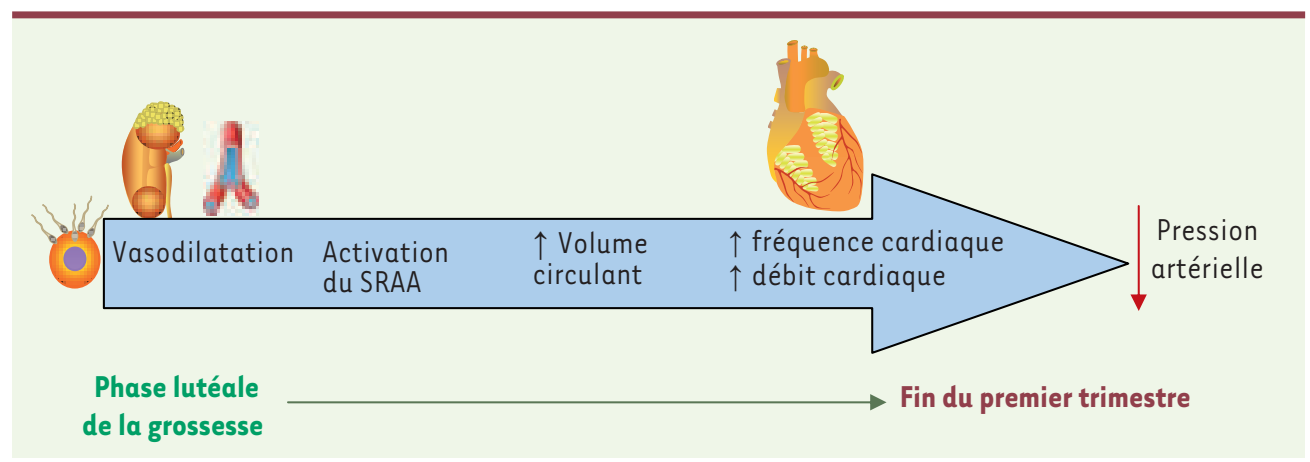

Figure 3. Schéma descriptif des événements hémodynamiques précoces présumés survenir au cours du premier trimestre de grossesse. Depuis la fécondation (à gauche) jusqu'à la baisse de pression artérielle, soit de la $8^{\mathrm{e}}-12^{\mathrm{e}}$ semaine, chez la femme. Notons que l'ensemble de ces phénomènes surviennent durant les 22 jours de gestation (sur 23) chez la rate. diminution du tonus vasculaire sont sans contredit les mécanismes qui causent la baisse de la résistance périphérique et, par conséquent, de la pression artérielle $[13,16]$. Mais ici encore, il y a un paradoxe puisque, en général, une stimulation du SRAA est associée à une augmentation du tonus vasculaire.

Une augmentation du SRAA par une diète faible en sodium (Figure 4, triangles jaunes) entraîne une sécrétion accrue d'aldostérone mais une diminution du volume sanguin, probablement à la suite d'une insuffisance rénale fonctionnelle, et la baisse de pression artérielle associée à la grossesse survient alors [19]. En revanche, si l'on inhibe le système (Figure 4, pentagone vert), on retrouve une augmentation du tonus vasculaire associée à l'absence de la baisse de la pression artérielle. Quels sont donc les mécanismes en jeu dans cette relation particulière entre les composantes du SRAA et la pression artérielle au cours de la grossesse? Nous estimons que la progestérone et l'œstradiol, ainsi que l'aldostérone, via le récepteur minéralocorticoïde, sont des joueurs clés (flèches pointillées, Figure 4). L'administration d'œstrogènes provoque une vasodilatation généralisée, une augmentation du débit cardiaque, une faible diminution de la pression artérielle et une réduction de la résistance systémique [25]. Cette situation ressemble étrangement à celle qui prévaut durant la gestation $[9,26]$. De plus, l'augmentation d'œstrogènes contribuerait à une augmentation de l'expression de la synthase endothéliale du monoxyde d'azote qui affecterait l'ensemble de la circulation sanguine [25].

\section{En guise de conclusion}

II semble que plus nous progressons dans la connaissance de l'adaptation de l'organisme féminin à la grossesse, plus nous constatons notre ignorance des étapes qui enclenchent cette adaptation. Les travaux de Salas et al. [6] et de Chapman et al. [7] nous ramènent aux observations de MacGillivray, et al. [27] et de Moutquin, et al. [28] montrant qu'en début de grossesse, on enregistre une chute de la pression artérielle, mais pas chez les femmes qui vont développer une prééclampsie. Nous suggérons que ces phénomènes 
se produisent encore plus tôt, soit juste après la conception, et que la phase lutéale de la grossesse, avant que le placenta ne prenne la relève du corps jaune de gestation, soit ici en cause. Si nous connaissons mal les phénomènes qui surviennent dès cette période, nous savons que l'échec de leur mise en place entraîne de sérieuses perturbations, par exemple, la mauvaise invasion trophoblastique des artères spiralées ou la dysfonction endothéliale, qui provoqueront à leur tour l'apparition des manifestations cliniques de l'hypertension gestationnelle et une restriction de la croissance intra-utérine. C'est ce champ de recherche qu'ont exploré ces auteurs $[6,7]$ et qu'il faudra beaucoup plus étudier. C'est aussi cette étape de la grossesse qui se prête bien aux expériences avec des animaux de laboratoire, en particulier la rate. Comme le notent Lindheimer et Sibai [29], au sujet de la prééclampsie: «...the disease is still mysterious,... Our limited resources should focus more on basic research, with human studies stressing prediction markers... ». C'est encore le défi qu'il nous revient de relever. $\diamond$

\section{SUMMARY}

The cardiovascular paradox of pregnancy

Despite widespread accessibility to prenatal care, little is known on the mechanisms initiating early maternal adaptation to pregnancy. Moreover, preeclampsia and intrauterine growth retardation remain the most frequent and serious complications of pregnancy. Recent studies, both in humans and in laboratory animals, have shown that very early events in gestation may be important determinants for the continuation of healthy pregnancy. Certain of these early adaptations appear to be linked to the corpus luteum of pregnancy, as ovarian steroid hormones (especially progesterone) would set the basic hemodynamic conditions, more specifically, generalized vasodilation. This new hemodynamic setup initiates a vicious cycle in which

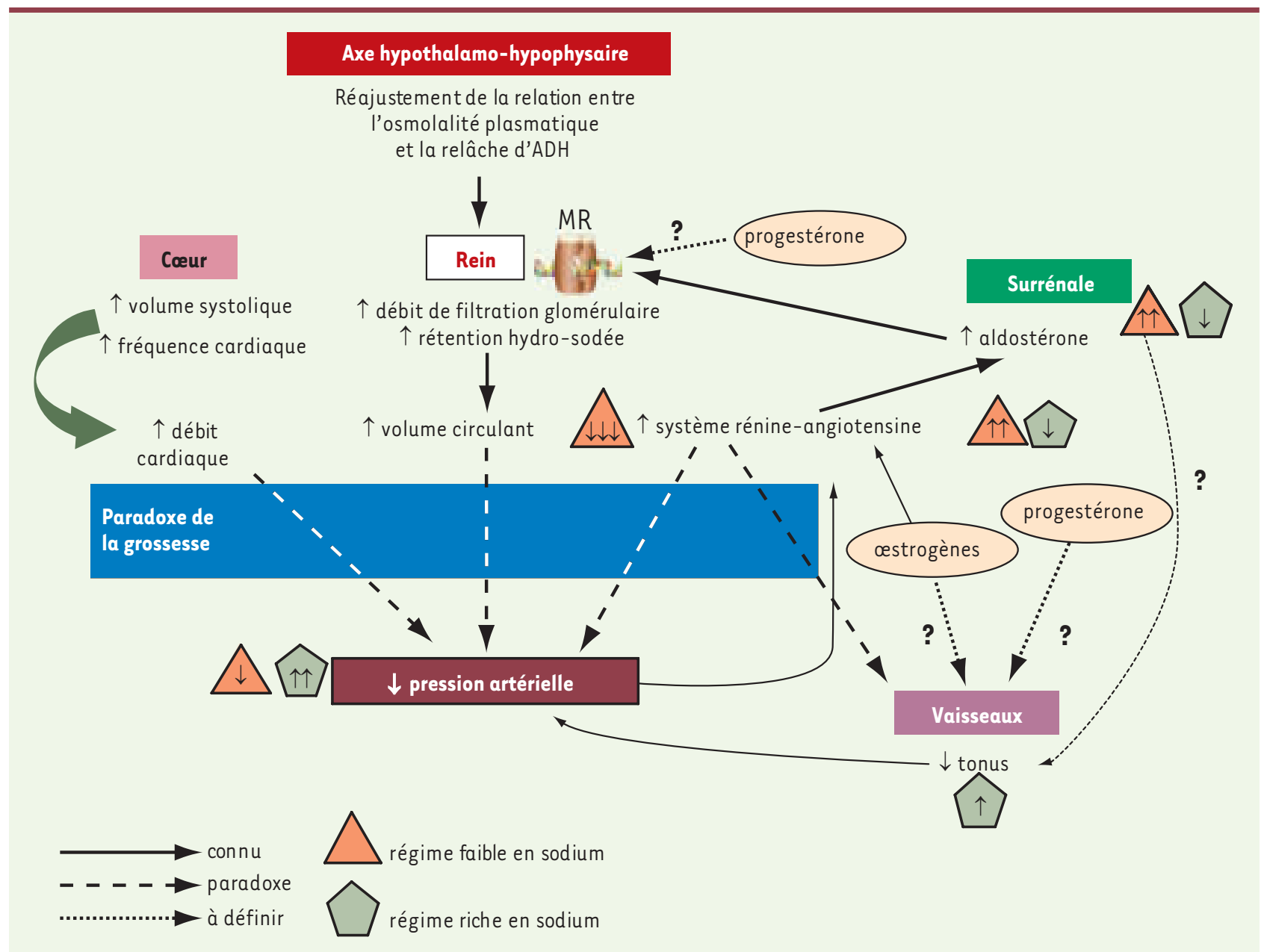

Figure 4. Description du concept du paradoxe cardiovasculaire de la grossesse. Celui-ci se présente comme une réponse inhabituelle de la pression artérielle aux principales manifestations de la grossesse (augmentations du SRAA, du volume sanguin circulant et du débit cardiaque). Les triangles jaunes et les pentagones verts représentent les données obtenues lorsque nous avons respectivement réduit et augmenté l'ingestion de sodium durant la dernière semaine (sur 3 ) de gestation chez la rate. On questionne le rôle des hormones stéroïdiennes dans ces processus. Bien que les données présentées aient été obtenues chez la rate, des observations similaires ont été faites chez la femme. 
the renin - angiotensin - aldosterone system is activated, together with the resetting of the control of antidiuretic hormone secretion relative to plasma osmolality. This leads to a gradual and substantial increase in plasma volume and a parallel increase in cardiac function (both heart rate and stroke volume) with the goal of maintaining blood pressure in the face of the generalised vasodilation. This includes the creation of a functional arterio-venous shunt represented by the utero-placental circulation. By the end of the first trimester, the decrease in peripheral vascular resistance is marked relative to the increase in cardiac output, resulting in a significant decrease in blood pressure that will be maintained until the third trimester. It is proposed that in preeclampsia, these very early events (vasodilation - increased plasma volume) fail to occur, resulting in an absence of the usual decrease in blood pressure, which is normally seen in the second trimester of pregnancy, and hypertension in the third trimester. Experimental animals, especially the rat, are suitable models to study this early maternal adaptation to pregnancy, since both endocrine and hemodynamic changes appear to be similar to humans. $\diamond$

\section{RÉFÉRENCES}

1. Dumont A, Merviel P, Berkane N, et al. Facteurs de risque de la pré-éclampsie. Presse Med $1999 ; 28: 2189-96$.

2. Lindheimer MD, Umans JG. Explaining and predicting preeclampsia. $N$ Engl J Med 2006 ; $355:$ 1056-8.

3. Poston L, Briley AL, Seed PT, et al. Vitamin C and vitamin $\varepsilon$ in pregnant women at risk for preeclampsia (VIP trial): randomised placebo-controlled trial. Lancet 2006; 367 : 1145-54.

4. Stepan H, Faber R, Dornhofer N, et al. New Insights into the Biology of Preeclampsia. Biol Reprod 2006 ; 74: 772-6.

5. Ianosi-Irimie M, Vu H, Withbred JM, et al. A rat model of preeclampsia. Clin Exp Hypertens $2005 ; 8: 605-17$.

6. Salas SP, Marshall G, Gutierrez BL, Rosso P. Time course of maternal plasma volume and hormonal changes in women with preeclampsia or fetal growth restriction. Hypertension $2006 ; 47: 203-8$.

7. Chapman AB, Abraham WT, Zamudio $S$, et al. Temporal relationships between hormonal and hemodynamic. changes in early human pregnancy. Kidney Int 1998 ; 54 : 2056-63.

8. Baylis C, Beinder $\varepsilon$, Suto T, August P. Recent insights into the roles of nitric oxide and reninangiotensin in the pathophysiology of preeclamptic pregnancy. Semin Nephrol 1998; $18: 208-30$.

9. St-Louis J, Massicotte G, Parent A. Effet anti-hypertenseur de la grossesse: influence de la réactivité vasculaire. Med Sci (Paris) $1988 ; 6: 358-65$.

10. Abdul-Karim R, Assali NS. Pressor response to angiotensin in pregnant and non pregnant women. Am J Obstet Gynecol 1961 ; 82 : 246-51.

11. Roy B, Sicotte B, Brochu M, St-Louis J. Effects of nifedipine and Bay K 8644 on myotropic responses in aortic rings of pregnant rats. Eur J Pharmacol $1995 ; 280: 1-9$.

12. Teeuw AH, De Jong W. Time course of decrease in blood pressure and in blood pressure response to vasopressor agents during pregnancy in the rat. Pflugers Arch 1973; $341: 197-208$.

13. Roy B, Sicotte B, Brochu M, St-Louis J. Modulation of calcium mobilization in aortic rings of pregnant rats: contribution of extracellular calcium and of voltage-operated calcium channels. Biol Reprod 1999; $60: 979-88$.
14. Brochu M, Gauvin JP, St-Louis J. Increase of aldosterone in adrenal cortex suspensions derived from pregnant rats. Proc Soc Exp Biol Med 1996; $212: 148-53$.

15. Simaan M, Cadorette C, Poterek M, et al. Calcium channels contribute to the decrease in blood pressure of pregnant rats. Am J Physiol Heart Circ Physiol 2002; 282 : H665-71.

16. Cadorette C, Sicotte B, Brochu M, St-Louis J. Effects of potassium channel modulators on myotropic responses of aortic rings of pregnant rats. Am J Physiol Heart Circ Physiol 2000; 278 : H567-76.

17. Keyes $L$, Rodman DM, Curran-Everett $D$, et al. Effect of $K_{\text {ATP }}^{+}$channe inhibition on total and regional vascular resistance in guinea pig pregnancy. Am J Physiol Heart Circ Physiol 1998 ; 275 : H680-8.

18. Meyer MC, Brayden JE, McLaughlin MK. Characteristics of vascular smooth muscle in the maternal resistance circulation during pregnancy in the rat. Am J Obstet Gynecol 1993; 169: 1510-6.

19. Roy-Clavel \&, Picard S, St-Louis J, Brochu M. Induction of uterine growth restriction with low-sodium diet fed to pregnant rats. Am J Obstet Gynecol 1999; $180: 608-13$.

20. Baylis C, Munger K. Persistance of maternal plasma volume expansion in midterm pregnant rats maintained on a zero sodium intake: evidence that early gestational volume expansion does not require sodium retension. Clin Exp Hypertens Pregnancy 1990 ; B9 : 237-47.

21. Beauséjour A, Auger K, St-Louis J, Brochu M. High-sodium intake prevents pregnancy-induced decrease of blood pressure in the rat. Am J Physiol Heart Circ Physiol 2003 ; 285 : H375-83.

22. Auger $\mathrm{K}$, Beauséjour A, Brochu M, St-Louis J. Increased $\mathrm{Na}^{+}$intake during gestation in rats is associated with enhanced vascular reactivity and alterations of $\mathrm{K}^{+}$and $\mathrm{Ca}^{++}$function. Am J Physiol Heart Circ Physiol 2004 ; 287 : H1848-56.

23. Mendelsohn ME, Karas RH. Molecular and cellular basis of cardiovascular gender differences. Science 2005 ; 308 : 1583-7.

24. Durr JA, Stamoutsos B, Lindheimer MD. Osmoregulation during pregnancy in the rat: evidence for resetting of threshold for vasopressin secretion during gestation. J Clin Invest $1981 ; 68: 337-46$.

25. Chen DB, Jia S, King AG, et al. Global protein expression profiling underlines reciprocal regulation of caveolin 1 and endothelial nitric oxide synthase expression in ovariectomized sheep uterine artery by estrogen/progesterone replacement therapyl. Biol Reprod 2006; $74: 832-8$.

26. Ahokas RA, Anderson GD, Lipshitz J. Cardiac output and uteroplacental blood flow diet-restricted and diet-repleted pregnant rats. Am J Obstet Gynecol 1983; 146: 6-13.

27. MacGillivray I, Rose GA, Rowe B. Blood pressure survey in pregnancy. Clin Sci $1969 ; 37: 395-407$.

28. Moutquin JM, Rainville C, Giroux L, et al. A prospective study of blood pressure in pregnancy: prediction of preeclampsia. Am J Obstet Gynecol $1985 ; 151: 191-6$.

29. Lindheimer MD, Sibai BM. Antioxidant supplementation in pre-eclampsia. Lancet 2006 ; 367: 1119-20.
TIRÉS À PART

J. St-Louis 